\title{
Cortical-hippocampal interactions and cognitive mapping: A hypothesis based on reintegration of the parietal and inferotemporal pathways for visual processing
}

\author{
B. L. MCNAUGHTON, B. LEONARD, and L. CHEN \\ University of Colorado, Boulder, Colorado
}

\begin{abstract}
Spatial navigation and the firing of hippocampal place cells can be driven as much by what an animal knows about its spatial world as by what it immediately experiences at a given location. If presented first with a set of spatially orienting cues, which are then removed during a test, navigation to a place of reward is accurate and place cells still fire in their correct locations despite the absence of the controlling cues (O'Keefe \& Speakman, 1987). Similarly, as reported here, place fields are disrupted in a familiar room in darkness if the animal is not shown its starting location, but remain intact in darkness if the starting location is known to the animal. A minimal computational model is presented to account for these results. The hypothesis proposes that conditional associations between places and movements are established during learning about an environment. Given a known starting location, these compound movement/place representations can be used to recall a sequence of target locations on the basis of the corresponding movement sequences alone. Recordings of posterior parietal neurons in rats performing a radial maze task reveal that this cortical region contains cells that are selective for specific combinations of environmental spatial features and motion states. The proposed model suggests how these compound movement/place representations could be combined with hippocampal spatial representations to account for the "blind" navigation phenomena described above. The model may also help us to understand the reasons for segregation of mammalian visual information processing into parietal and inferotemporal streams as described by Ungerleider and Mishkin (1982).
\end{abstract}

Ungerleider and Mishkin (1982) and others have proposed that, in the mammalian visual system, extrastriate visual processing is segregated into two distinct, polysynaptic functional pathways. One of these, the "inferotemporal" pathway, which in primates culminates in Area TE of the temporal pole, appears to be specialized for the formation of high-level mnemonic representations of objects or complex conjunctions of visual stimuli. This idea is based on observations that single units in this region respond best to complex visual stimuli, being much more affected by form, texture, or color than by location, orientation, or movement (e.g., Gross, Bruce, Desmoine, Fleming, \& Gattass, 1981), and that lesions of this area produce severe deficits in object-recognition memory (Cowey \& Gross, 1979; Mishkin, 1982). On the other hand, the "parietal" pathway, culminating in Posterior Parietal Area 7 (PG, PF), appears somehow to be involved in representing the spatial location of objects. This conclusion is based on the well-known syndrome following Area 7 damage involving spatial neglect, deficits in hand-eye coordination, and spatial-memory impairment, and by the fact that single units in this region are not very sensitive to the form of visual stimuli, but respond to their location in space (Andersen, Essick, \& Siegel, 1985), their

Correspondence may be addressed to B. L. McNaughton, Department of Psychology, University of Colorado, Boulder, CO 80309.
3-D movement in space (Sakata, Shibutani, Kawano, \& Harrington, 1985; Tanaka et al., 1986), the movements of the animal's eyes or limbs (Hyverinen, 1982; Mountcastle, Lynch, Georgopoulos, Sakata, \& Acuna, 1975), or to the optic flow patterns typically generated by the animal's own movement in space (Motter \& Mountcastle, 1981; Motter, Steinmetz, Duffy, \& Mountcastle, 1987). One interesting feature of neurons in the parietal pathway is their tendency to respond to specific sensorysensory or sensory-motor conjunctions (Andersen, 1987).

Ungerleider and Mishkin (1982) left open questions of how and where these two processing streams might be reintegrated. They suggested that one likely location is the hippocampal formation which, as illustrated in Figure 1, is one site of reconvergence of the two visual streams. As proposed by Smith and Milner (1981), the hippocampus might thus enable the rapid memorization of particular locations occupied by particular objects, a function not too dissimilar from O'Keefe and Nadel's (1978) proposal that the hippocampus forms the neuronal substrate for a nonegocentric cognitive map of spatially extended environments. The present hypothesis concerns an attempt at a first-order description of how this reintegration may take place.

Visuospatial behavior and memory in primates are extremely complex phenomena. This complexity is exacerbated by the fact that these operations are subserved by 


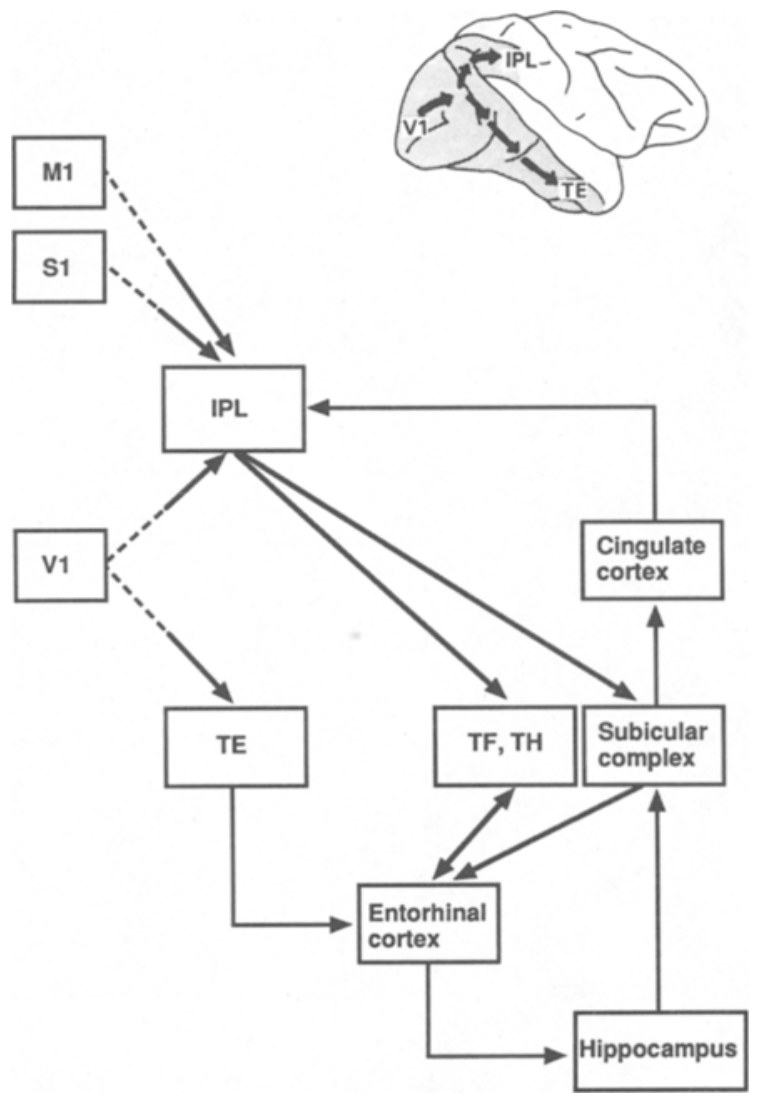

Figure 1. Simplified illustration of the reconvergence of the parietal and inferotemporal visual streams in the hippocampal formation. (Inset adapted from Mishkin, Ungerleider, \& Macko, 1983.)

a major proportion of the primate cortical mantle, involving numerous highly differentiated subareas whose detailed anatomy and physiology are, at best, poorly understood. Furthermore, in the primate, a coordinate transformation is required because of the major role played by the voluntary saccadic eye movement and smooth pursuit systems. These movements of the eye in the orbit dissociate the retinal coordinates from the head/body axis for most visual behaviors, and this dissociation must be accounted for by other motor systems. Finally, technical considerations have precluded extensive study of neural activity in unrestrained primates in relation to behavior in spatially extended environments (i.e., cognitive mapping). Recent studies in rodents (Foster, Castro, \& McNaughton, 1988, 1989) have shown that spatially selective firing of hippocampal cells is totally abolished by head and/or body restraint. Such complexities make the prospect of developing testable computational models of spatial representation in the primate intimidating, to say the least.

On the other hand, the voluntary saccade system of rodents is poorly developed (Lashley, 1932), and orientation to visual stimuli is accomplished primarily by reorientation of the head/body axis. Allocentric visuospatial behavior and learning in the rodent, being considerably less differentiated and complex than in the primate, are quite well understood, at least phenomenologically (see Leonard \& McNaughton, in press, for a recent review). Furthermore, lesion studies over the past decade or so have established beyond question that the hippocampus plays a crucial role at least in the initial formation of spatial representations of the world (see Barnes, 1988, for a recent review). Finally, the ability to study single-unit activity in rodents moving freely in extended environments has led to the finding that the firing patterns of pyramidal cells in the rodent hippocampus are dominated by spatial stimuli in its environment, particularly visual ones (Hill, 1978; McNaughton, Barnes, \& O'Keefe, 1983; Muller, Kubie, \& Ranck, 1987; O'Keefe, 1976; O’Keefe \& Dostrovsky, 1971; Olton, Branch, \& Best, 1978). Without doubt, the most important finding in this field has been the demonstration by O'Keefe and Speakman (1987) that this spatial firing can be driven as much by what the animal "knows" about the spatial relationships of its world as by what the animal actually experiences at a given location.

The O'Keefe and Speakman (1987) experiment was based on earlier behavioral work of O'Keefe and Conway (1980), which showed essentially that, once shown their initial location relative to a controlled set of distal spatial cues which completely determined their spatial behavior, rats could remember this information and use it to guide their navigational behavior accurately after the complete removal of the relevant controlled cue set. O'Keefe and Speakman showed that under these conditions, hippocampal "place cells" fired in their correct locations relative to the previously available cue set even though these cues were not physically present.

In the following, we present a verification of the O'Keefe and Speakman (1987) result using a different procedure (Leonard, McNaughton, \& Barnes, 1985) from which a few additional insights may be gained. We then develop a minimal computational model to attempt to account for these findings. The model is based on the simple idea that the metric by which spatial relationships are encoded is the elemental movement required by the animal to bring two different "local views" of the environment sequentially into register on its sensorium (McNaughton, 1987). The components of the model bear some resemblance to the segregation of visual processing into the two functional streams described above. We then describe recent results from single-unit recording from rat sensorimotor and posterior parietal cortices which provide some support for this idea.

\section{INTERNAL REPRESENTATION OF ORIENTATION IN HIPPOCAMPUS}

The following experiment was carried out once with each of 2 rats. The animals were thoroughly trained to perform the well-known eight-arm radial-maze problem, 
A

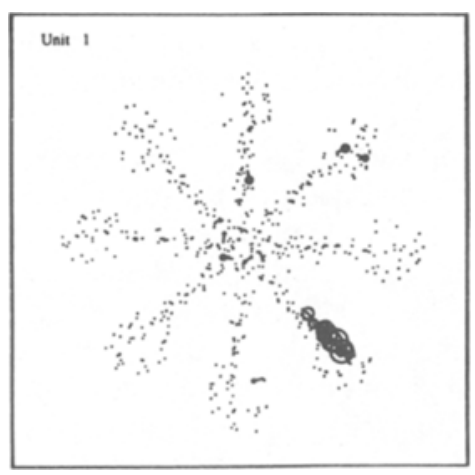

B

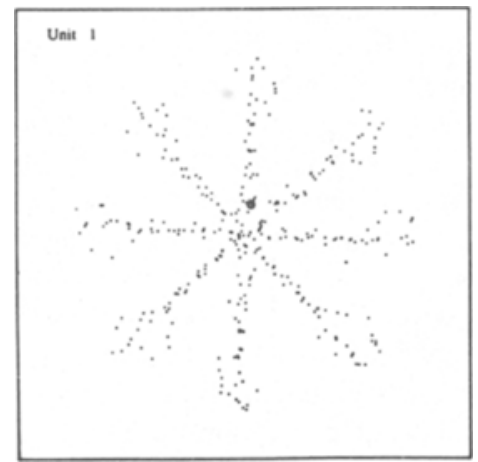

C

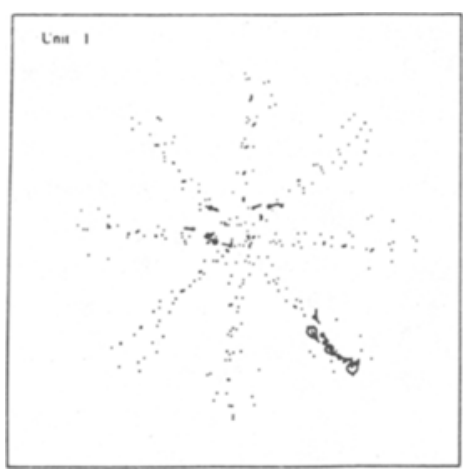

D

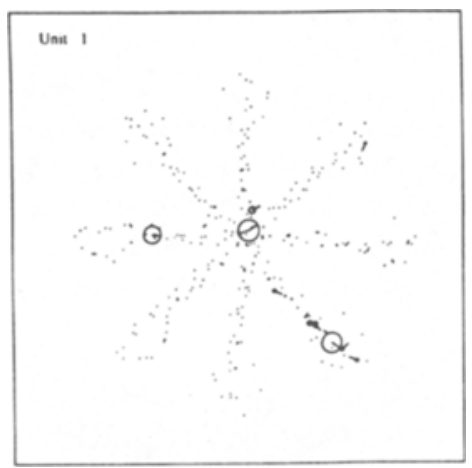

previous day

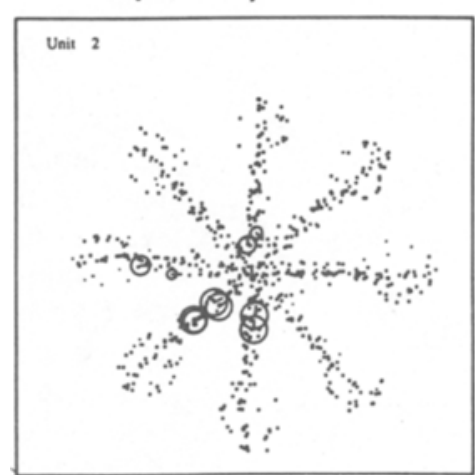

PHASE 1 lights out in rats absence

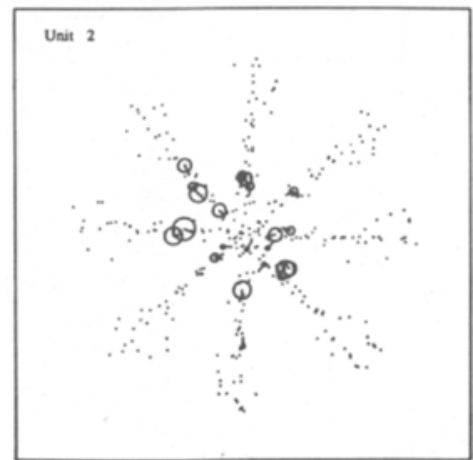

PHASE 2 lights on

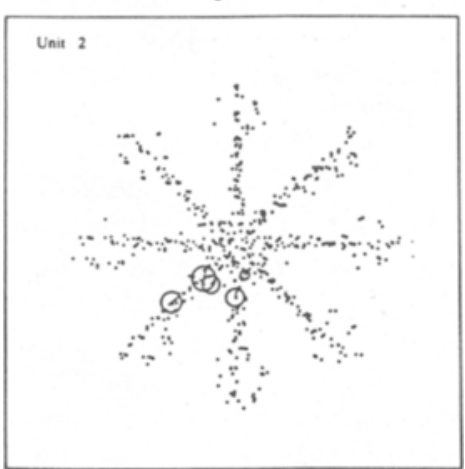

PHASE 3 lights out in rat's presencs

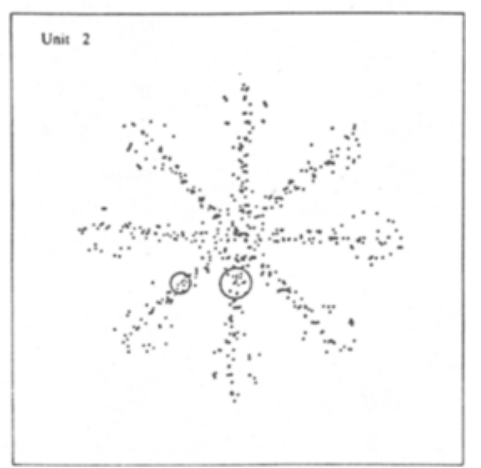

Unin 3
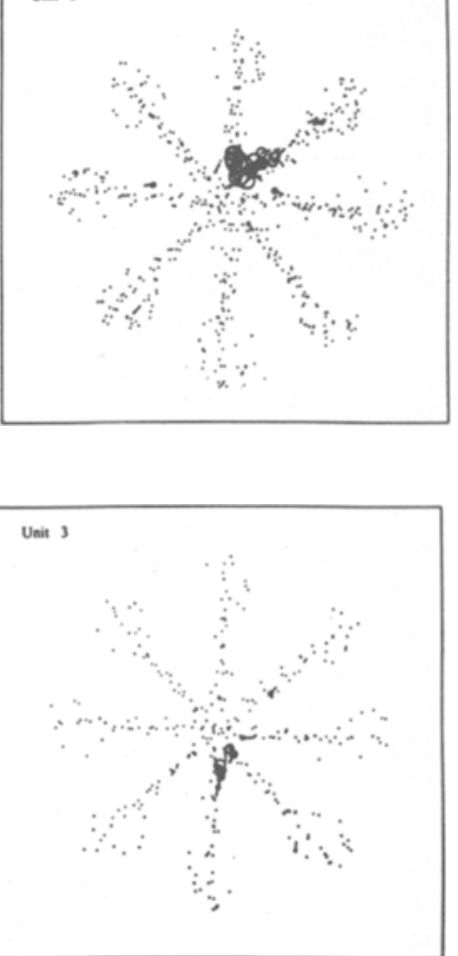

Uaus 3

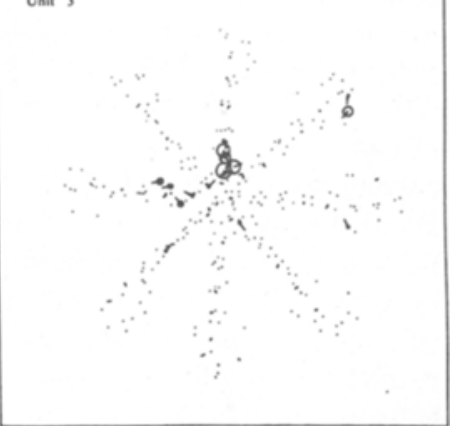

Unit 3

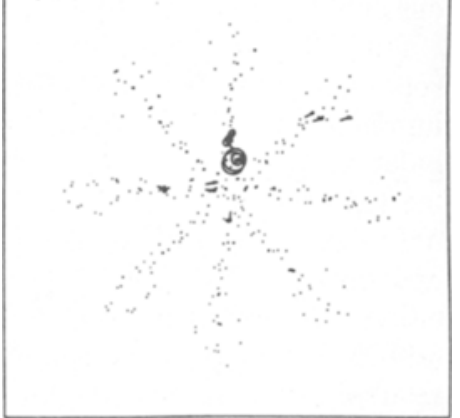

Figure 2. Place fields of three simultaneously recorded complex-spike cells on the eight-arm radial maze before and during the darklight-dark experiment (see text for details). Extracellular single-unit recordings were obtained while simultaneously tracking the spatial position of the rat. The area of the plotted circle is directly proportional to the locally computed firing rate. Overlapping circles indicate that the cell fired on repeated traverses through the region. Dots indicate that the animal traversed a region but the cell did not fire. The dashed lines originating at the circle centers indicate the direction of motion of the animal. In general, knowledge of starting orientation was sufficient to preserve the spatial selectivity of units in darkness. 
in which a food reward is obtained from the end of each of the eight arms. The maze was located in a $12 \times 12 \mathrm{ft}$ room illuminated by a $40-\mathrm{W}$ lamp on the south wall, with numerous other visual cues distributed around the room. This task can be conceptualized as imposing a polar coordinate system on the animal's behavior. Radial information is available to the animal largely from the local geometry of the arms themselves. Because the arms are identical to one another, the angular coordinate is specified solely by the distal visual cues. The animal can tell its radial distance from the center, in the absence of visual information, through its somatosensory system and probably some integration of its own motor output (Mittelstaedt \& Mittelstaedt, 1980).

Using the "stereotrode" recording method (McNaughton, O'Keefe, \& Barnes, 1983), pyramidal cells from CA3/4 were isolated. First, the spatial firing of cells was characterized (see Figure 2) under standard illumination conditions and was shown to be stable over at least several days. On the experimental day, the animal was brought from the colony room in an enclosed box and was introduced to the apparatus in total darkness. The animals were able to perform the task under this condition, and their positions could be tracked and recorded in the normal fashion by monitoring the infrared-emitting diode mounted on the animals' heads.

During this first dark phase, the spatial selectivity of firing was disrupted. For most cells, the disruption was restricted to the angular coordinates. The radial component was preserved, in that the firing remained at the correct distance from the end of the arm. For 1 animal, in which three cells were simultaneously recorded, one cell changed its field to a different arm, one cell fired at the correct radial position but on several arms, and one cell stopped firing altogether. For the single cell recorded in the second animal, the field changed to the incorrect arm (angular coordinate). These data suggest that when there is confusion about the angular coordinate, different cells respond in different ways in the same experiment. Some cells may develop independent "hypotheses" about the correct orientation. Moreover, it is clear that both the local geometric features of the apparatus and the distal visual cues contribute to the spatially selective firing.

Phase 2 of the experiment consisted of turning on the room light and running additional trials under normal illumination. During this phase, the correct radial and angular firing was observed. Phase 3 involved turning out the light again, and running additional dark trials. The only difference between Phase 3 and Phase 1 was that now the animal presumably knew its initial angular orientation. As predicted from the O'Keefe and Speakman (1987) results, information about the starting orientation relative to the distal cues was sufficient for the correct spatial (angular and radial) firing to be maintained in total darkness, at least for the first several trials.

We believe that the most plausible interpretation of these and O'Keefe and Speakman's (1987) results is that, in highly familiar environments in which the animal knows its starting orientation, the animal is able to recall internal representations of the spatial consequences of its own specific movements. We next present a specific hypothesis about the neural circuitry that might accomplish this.

\section{CONDITIONAL ASSOCIATIONS AMONG PLACES AND MOVEMENTS}

Perhaps the simplest hypothesis that might account for the results described above is that, during exploration, the spatial relationship between adjacent locations is encoded in terms of the elementary movement that connects them. For example, if the animal makes a left turn to get from Location A to Location B, it might learn to recall the local features of Location $B$ the next time it finds itself in Location A simply by making (or imagining) a left turn. Such learning has the form of a conditional association between the representation of a left turn and its consequent representation of Location $B$. The association is conditional because not all left turns lead to Location B, only those made in the context of being in Location $A$. This is illustrated schematically in Figure 3.

Our thinking about how neurons might implement such conditional associations is essentially an extension of the ideas of Hebb (1949), Steinbuch (1961), Willshaw, Buneman, and Longuet-Higgins (1969), Marr (1969, 1971), and Kohonen (1972) about how simple associations are formed (see McNaughton \& Morris, 1987, for a recent discussion of how these ideas relate to the circuitry and processes found in the hippocampus). The essence of these ideas is illustrated in Figure 4a. Storage of an association can be thought of as the formation of the outer product

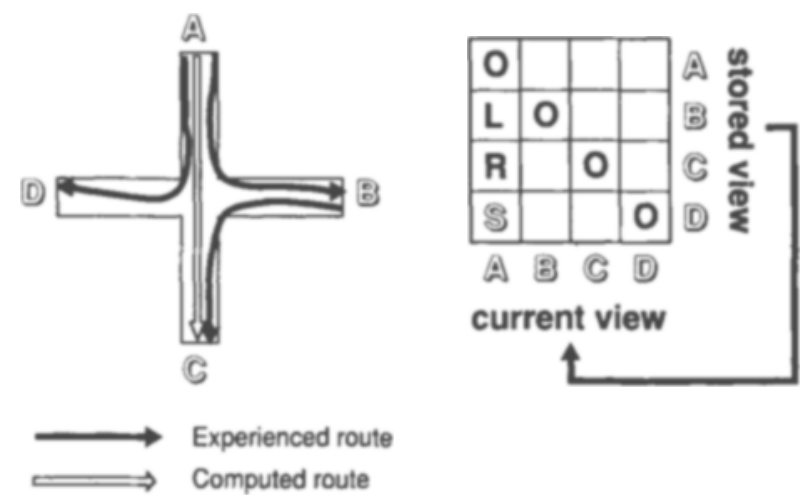

MOVEMENTS: L-left, R-right, S-straight (computed)

Figure 3. Ilustration of the transition matrix concept of place learning. Imagine that a rat runs on a " + " maze with locations specified by four distinguishable landmarks, A, B, C, and D. Ignoring, for the moment, that the animal must make $180^{\circ}$ turns at the arm ends, learning about this environment involves "filling in" a square matrix, whose axes correspond to locations, with the specific movements (Left, Right, Straight) linking these locations. The location information need not be more complicated than a representation of the "local view" of the environment (McNaughton, 1987, 1989). 
matrix of two binary representation vectors $(X, Y)$. Recall of one vector of a pair results from multiplying the matrix by the other vector, followed by a normalization operation. The latter operation involves integer division of the vector-matrix inner product by the sum of the components (the number of $1 \mathrm{~s}$ ) of the input vector. This operation, which is responsible for both correct paired-associate recall and the ability to reconstruct the correct output from a fragment of the corresponding input, is explained further below. Within limits, a number of event pairs can be simply overlaid in a single matrix in such a way that the individual paired associates can still be extracted reliably using this recall procedure.

Marr $(1969,1971)$ made the first clear proposals as to how these formal mechanisms might be implemented in known neuroanatomical systems. Figure 4 is largely abstracted from this work and represents one of several possible variations on Marr's theme. Elsewhere (McNaughton \& Nadel, 1989) it has been proposed that such networks might appropriately be referred to as Hebb-Marr

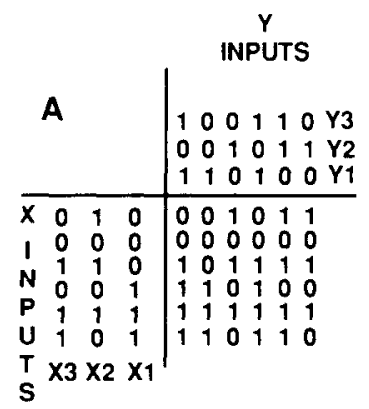

CORAECT RECALL

(Recall of Y3 given $X 3$ )

$001011=X 3$

$\mathrm{X} 3 \cdot \mathrm{C}=322332$

$322332 / 3=100100=Y 3$

PATTERN COMPLETION

(Recall of Y3 given a part of $X 3$ )

001001 is a subset of $X 3$

$001001 \cdot \mathrm{C}=\mathbf{2 1 1 2 2 1}$

$211221 / 2=100110=Y 3$
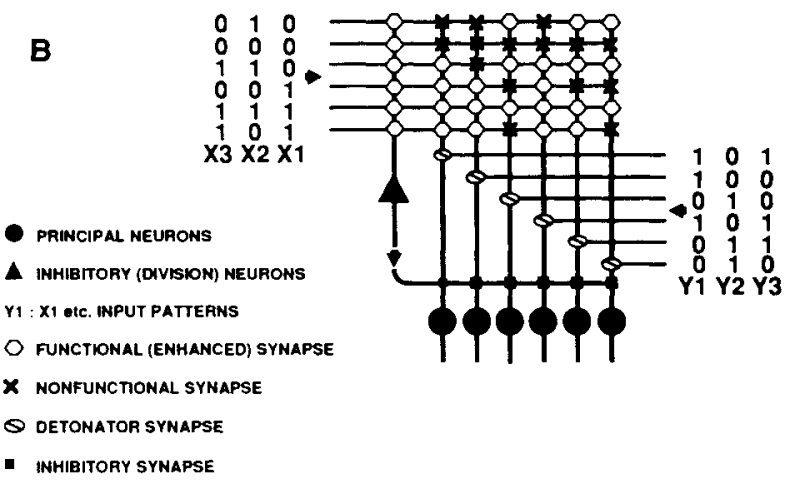

Figure 4. (A) The formal principles of distributed associative memory. Events are represented as input vector pairs (e.g., X1, Y1). Associations among the elements of the events are stored in a simple matrix ( $C=c_{i j}$, which has entries of 1 at all locations where two $1 s$ in the current event pair (or previous pairs) have intersected. Recall of a $Y$ pattern given only the corresponding $X$ pattern is accomplished by multiplying the rows of $C$ by the corresponding $X$ element, summing the columns, and then (integer) dividing the elements of the resulting vector by the number of $1 \mathrm{~s}$ used for recall (see example). (B) One version of the Hebb-Marr model for how these formal principles might be implemented in neural circuitry. See text for details on how this circuit operates. (Adapted from McNaughton \& Morris, 1987.) nets, because they incorporate essential ideas of both of these pioneers.

Minimal Hebb-Marr nets consist of three or four classes of neurons coupled in specific ways by at least three different kinds of synapse. In Figure $4 b$, for example, there is a population of "principal cells" whose output state is to represent the desired event. The principal cells receive and integrate three types of input from other neurons. The representation to be stored is conveyed via one input ( $Y$ in Figure 4b) that is connected in a one-to-one fashion with the principal cells via powerful synaptic contacts ("detonator synapses"). The strength of these connections is sufficient to ensure that the input is mapped exactly onto the output of the principal cells. A second "contextual" input ( $X$ in Figure $4 b$ ) is connected exhaustively with the principal cells via "Hebb" synapses. Hebb synapses are initially nonfunctional, but can be modified or enhanced to some fixed strength according to the simplest variant of Hebb's rule: if and only if the presynaptic and postsynaptic elements are simultaneously active, the connection undergoes an irreversible transition from its ineffective to its effective form. The synaptic weights ( 0 or 1 ) of active inputs of the $X$ type summate linearly on the principal cell to determine its activation state. This input constitutes the context in which the corresponding $Y$ event is to be recalled.

The third input comes from an inhibitory interneuron. The physiology of inhibitory synapses is assumed to be such that they essentially divide the activation of each principal neuron by a term proportional to the number of elements of the $X$ (context) path that are active during a particular recall cycle (i.e., the number of $1 \mathrm{~s}$ in the input vector). The reason for this is very simple. The objective is to perform paired-associate learning for multiple pairs. We "train" the system with various events (the $Y$ inputs) presented in specific contexts (the $X$ inputs). Later, we probe the system with a context. The response should be a representation of the corresponding event. In order to discover whether the current contextual $(X)$ input is one to which a given unit has been trained to respond, each unit in the layer must determine whether all of its currently active inputs have been involved in at least one previous event-context pair. If so, then it should produce output. If not, then the current input pattern must not belong to the set of contexts to which the unit has been trained to respond, and the unit should remain silent. Note that, to the extent to which previous inputs were similar (i.e., shared some common elements), some of a unit's inputs will likely have been modified by other patterns. The simplest solution (which, as is often the case, turns out to be the most biologically plausible one) is to add up the total number of elements of the input that are currently active, and divide the activation on each unit in the layer by this amount. If the unit should respond, the result will be 1 because all of the active synapses will have been enhanced. If not, the result will be less than 1 . Note that 
with this formalism, the complete context is not necessary; all that is required is a fragment sufficiently large to distinguish it from other contexts. It turns out that the properties of feed-forward inhibitory interneurons and synaptic modification in the hippocampal formation conform rather well to the requirements of this scheme (see McNaughton \& Nadel, 1989).

So far, we have described a plausible neural network for simple associative memory. Converting this to a conditional associative memory of the form postulated to underlie spatial representation requires only a minor modification. The basic idea is illustrated in Figure 5, which depicts several different sets of neurons. One set generates representations of the animal's movements. In the simplest case, these cells should discriminate among three modes of motion: forward motion, left turns, and right turns. Another set of cells generates representations of the conjunctions of visual features (objects)in any particular local view of the environment. These are the representations we desire to recall on the basis of some combination of previous location and movement. We therefore project these as the primary "detonator" input to a Hebb-Marr network of the sort just described.

We now take the output of the Hebb-Marr network and combine it with movement information by projecting both onto an additional set of neurons. In this way we can generate a population whose output represents specific conjunctions of visual features and elementary movements. We use these conjunctive representations as the contextual input to the Hebb-Marr association net. The context in which a particular local representation of the environment occurs is thus the specific conjunction of a previous local representation and an elementary movement. Once learned, these conjunctions alone should be enough to elicit recall of the corresponding local representation. In the context of typical primate experiments, we might express this as, "Given Object $A$ is on the fovea, Object B will be foveated by shifting the gaze through an angle, q." Assuming the environment has been reasonably well explored, chains of local representations

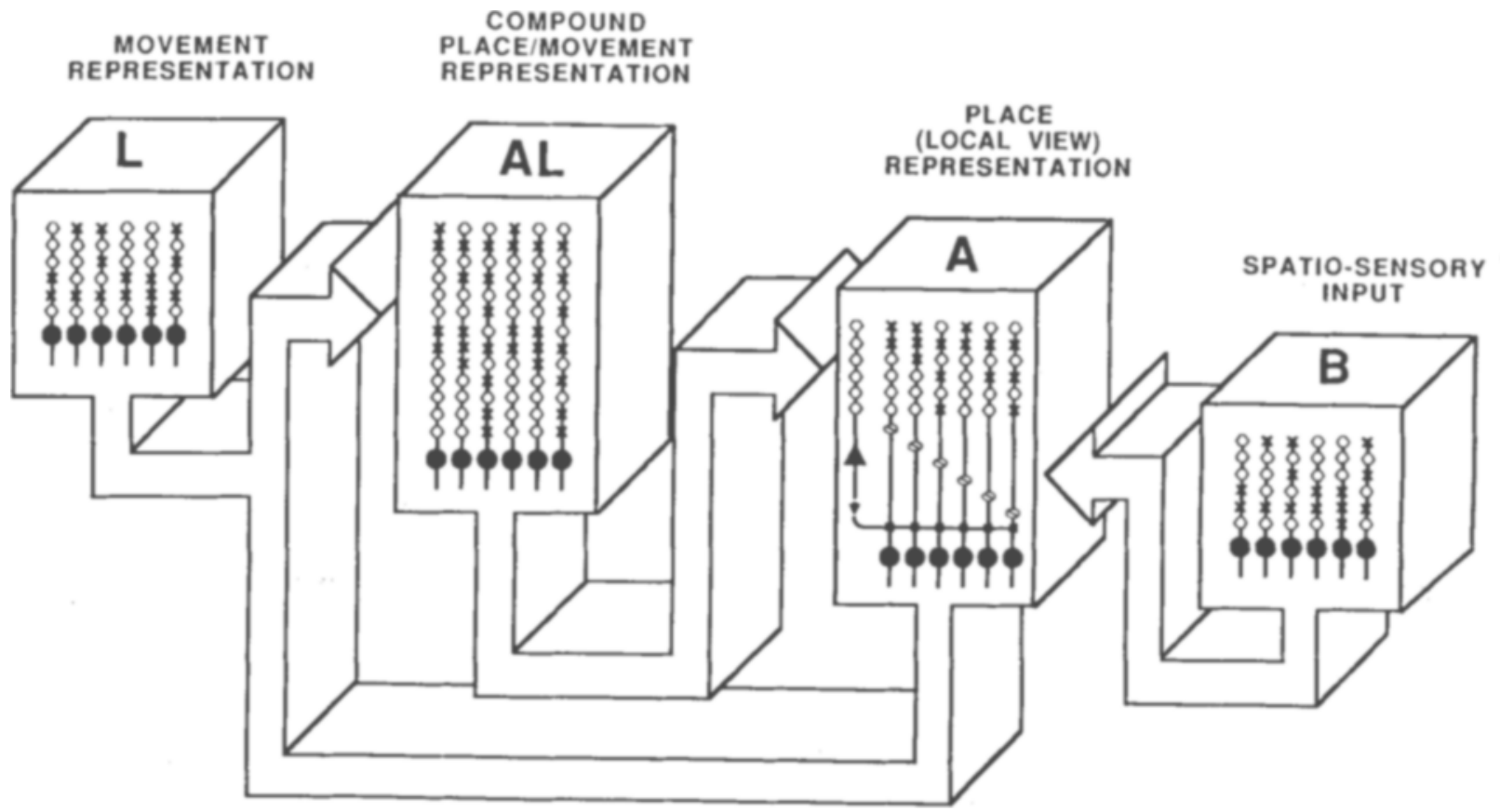

Figure 5. A simple model for implementing the transition matrix idea for conditional associations of local view representations with movements. In essence, a compound representation for specific combinations of locations and movements is generated. These event representations can be produced by combining the output of a heteroassociative matrix representing the local features of the world (e.g., Position A) with the output of the movement-representation system (e.g., Left) in a third set of neurons. This could produce a system whose output represents unique combinations of local view and movement (e.g., AL). Projecting this information back into the beteroassociative network via modifiable synapses would permit the current local view (e.g., Position B) to be associated with whatever combinations of view and movement preceded it. Subsequently, that representation (i.e., Position B) could be recalled whenever an appropriate compound place/movement representation (i.e., $\mathrm{AL}$ ) was presented. The system would then be capable of internally generating representations of sequences of locations on the basis of movement input alone. Note that only the starting location needs to be specified. A system such as this could account for the finding that hippocampal place cells fire in their correct location in darkness provided that the animal is informed about its starting location (see Figure 2). [From "Neuronal mechanisms for spatial computation and information storage," by B. L. McNaughton, 1989, in L. Nadel, L. A. Cooper, P. Culicover, and R. Harnish (Eds.), Neural connections and mental computations (pp. 285-349), Cambridge, MA: MIT Press. Copyright 1989 by MIT Press. Reprinted by permission.] 
could be sequentially recalled using only information about the starting location and the sequence of movements. This would provide a sufficient explanation for the results of O'Keefe and Speakman (1987) and those presented above. Moreover, the same formal structure might serve as a general model for the learning of conditional relationships between actions in a given sensory-motor context and the consequences of these actions.

From this model, then, one might conjecture that the inferotemporal visual pathway conveys high-level information about what objects are present in the visual scene, and in this sense characterizes the current location. On the other hand, the parietal pathway should convey representations of specific conjunctions of motion and location (see Figure 5).

\section{RAT PARIETAL CORTEX DURING SPATIALLY EXTENDED BEHAVIOR}

Kreig's Area 7 in the rat corresponds roughly to the primate posterior parietal region, although the homology is not perfect and the cytoarchitectural boundaries are

\section{LEFT TURN}

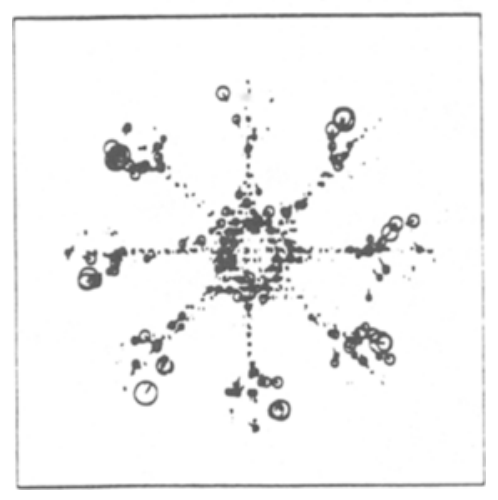

LEFT TURNS AT ENDS OF 'ARMS

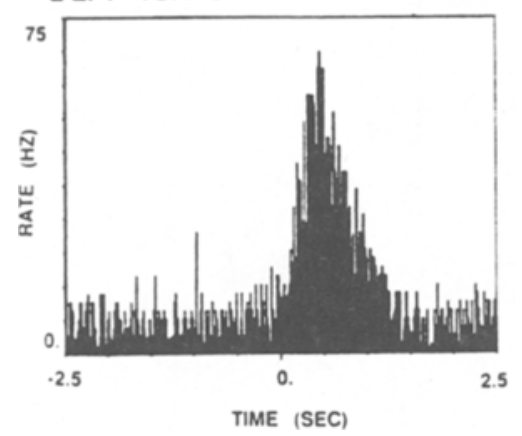

RIGHT TURNS AT ENDS OF ARMS

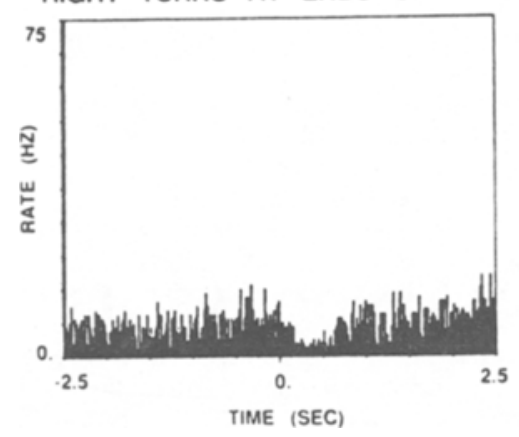

RIGHT TURN

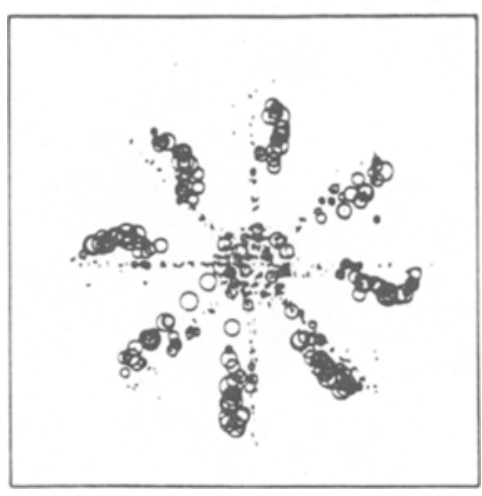

LEFT TURNS AT ENDS OF ARMS

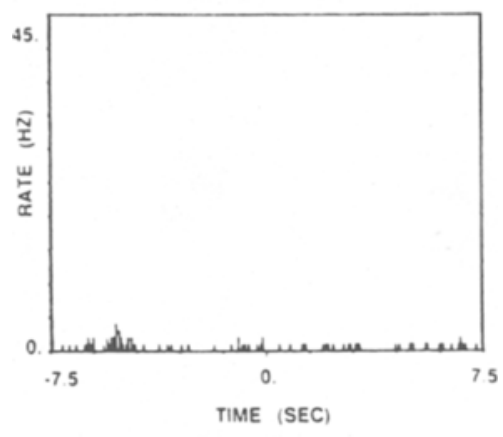

RIGHT TURNS AT ENDS OF ARMS

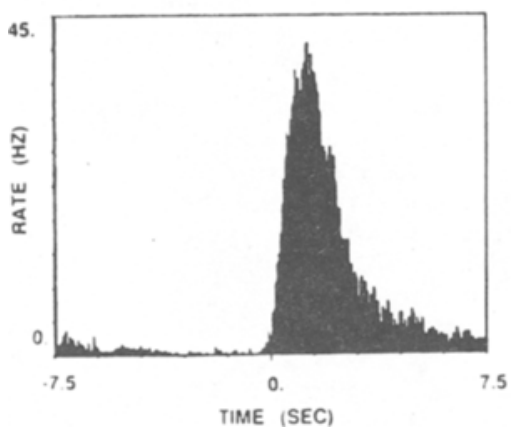

\section{STRAIGHT AHEAD}

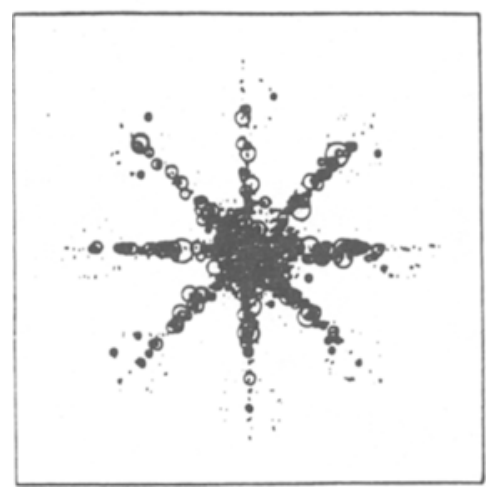

REACHED ENDS OF ARMS

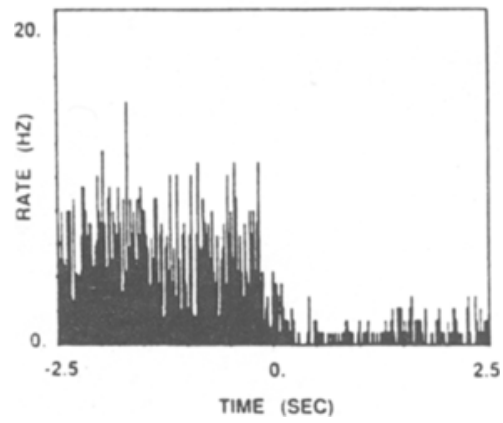

STARTS INBOUND AFTER RIGHT AND LEFT TURNS

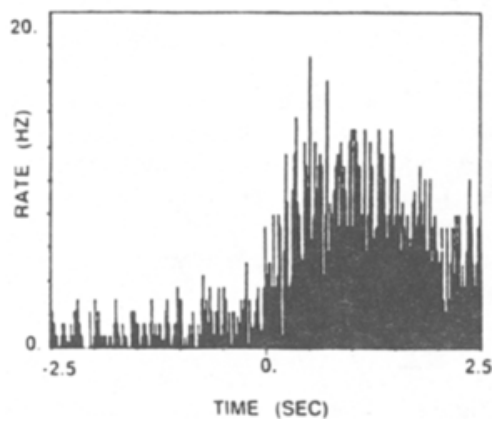

Figure 6. Behavioral correlates of three different neurons recorded in the sensorimotor cortex of a freely behaving rat. Cells were recorded while the animals ran the eight-arm radial-maze task. A substantial proportion of cells in this region can be roughly characterized as selective for a specific direction of turn or for forward motion. This selectivity appears to be independent of where the movement is executed. (Adapted from McNaughton, 1989.) 
somewhat vague. It is reciprocally connected with the lateral posterior thalamus (the rodent homologue of the pulvinar), the secondary visual cortex, the sensorimotor cortex, and the dorsal bank of the rhinal sulcus.

Over the past several years, we have been studying the activity of cells both in this region and in the sensorimotor overlap zone adjacent to it while animals traverse the radial maze (Chen \& McNaughton, 1988; McNaughton, Green, \& Mizumori, 1986). In the sensorimotor overlap zone, we found that a significant proportion of cells were selectively active during particular modes of motion that could be roughly characterized as left turns, right turns, and forward motion. This activity was essentially independent of the location on the maze where the behaviors occurred. Examples of such selectivity are shown in Figure 6.

In the parietal region, many cells are similarly selective for specific modes of movement. In addition,
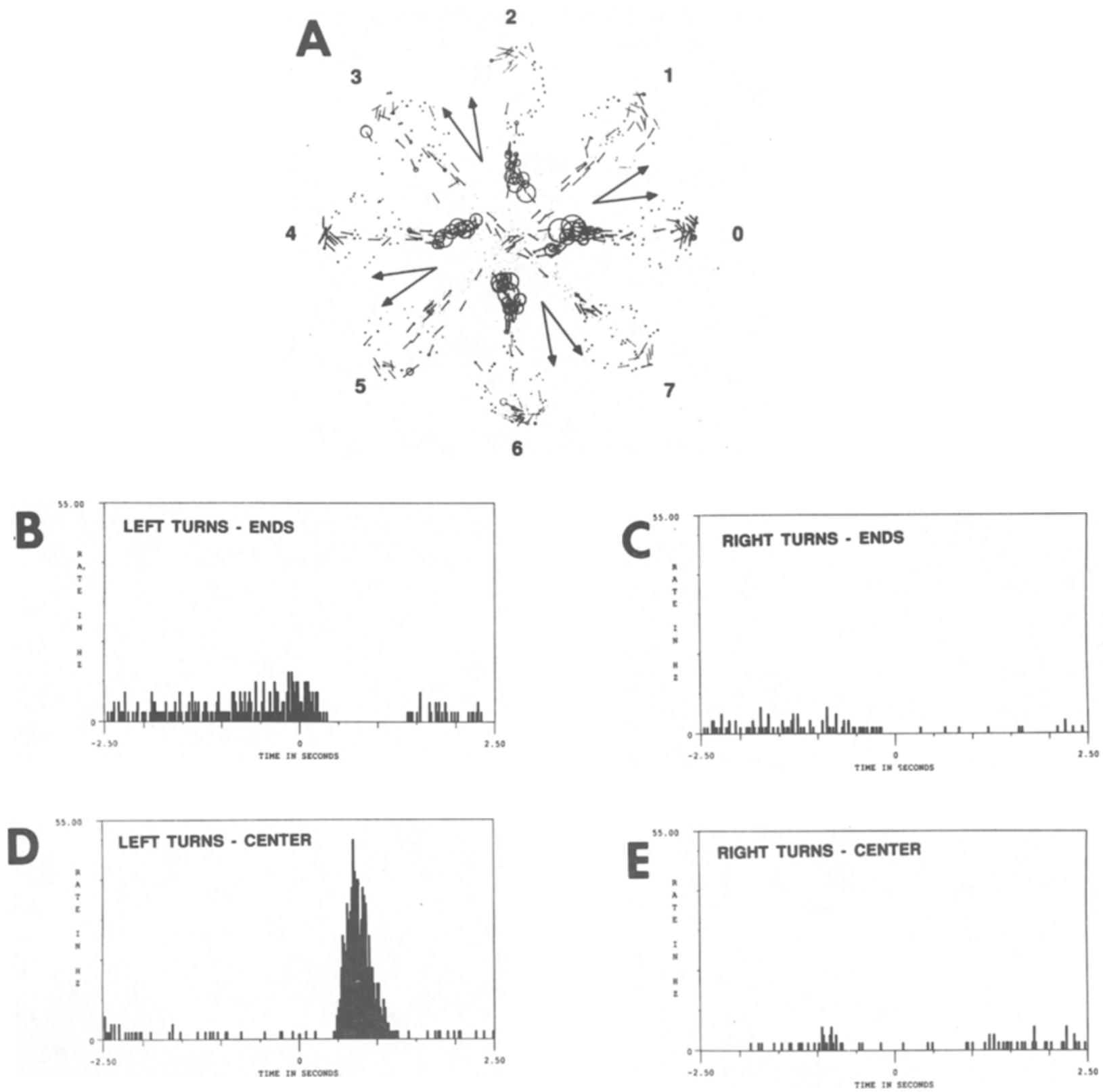

Figure 7. Illustration of a rat parietal neuron which fired selectively during left turns at the maze center, but not during left turns at the arm ends. To demonstrate this, the animal was required simply to run back and forth between pairs of adjacent arms (arrows in $\mathbf{A})$. Corresponding event histograms are shown in $B-E$. 
however, about $22 \%$ of parietal cells exhibited some combination of either movement selectivity and visual response, or movement selectivity in conjunction with local features of the maze. For example, one cell was selectively active during right turns, but only if there was light in the lower nasal retina. This resulted in a place $\times$ movement conjunction because of the discrete light source in the environment. Another cell was selectively active during left turns at the maze center, but not during turns at arm ends (Figure 7). Some cells were selective for a particular turn direction but only on some maze arms (Figure 8). Others were highly active during forward motion in one radial direction but were much less active in the other (Figure 9). One cell responded during radially outward movement toward the room light and followed changes in the location of the light. In darkness, however, it fired selectively in relation to the remembered location of the light.

We suggest that, in the rat, such conjunctive coding of movement and spatial location may provide one essential component of a larger system that is organized for the generation of representations of spatial relationships. Hopefully, the same conceptual framework may provide some insight into the role of the more complex forms of sensorimotor conjunctions represented in the parietal cortex of primates.
A

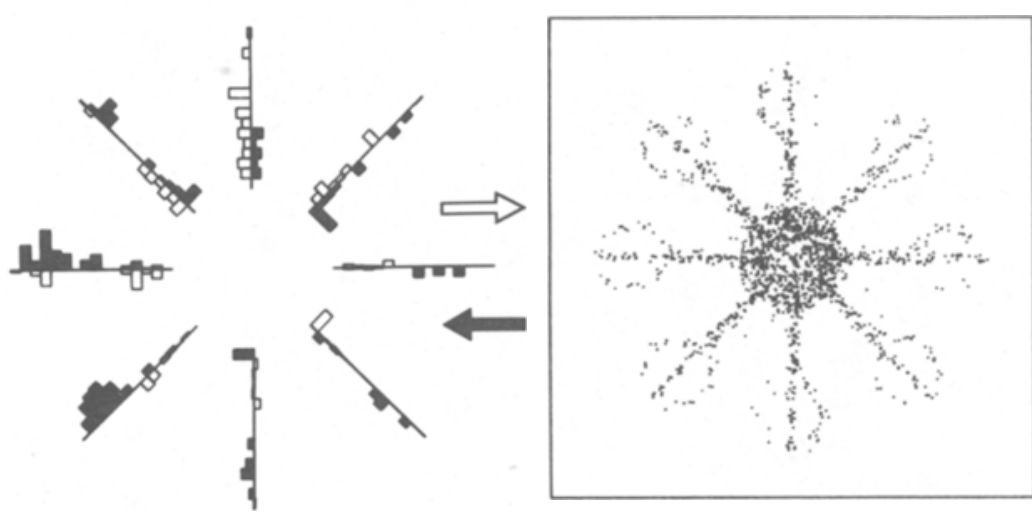

RIGHT TURNS

AT 3 WESTERN ARMS
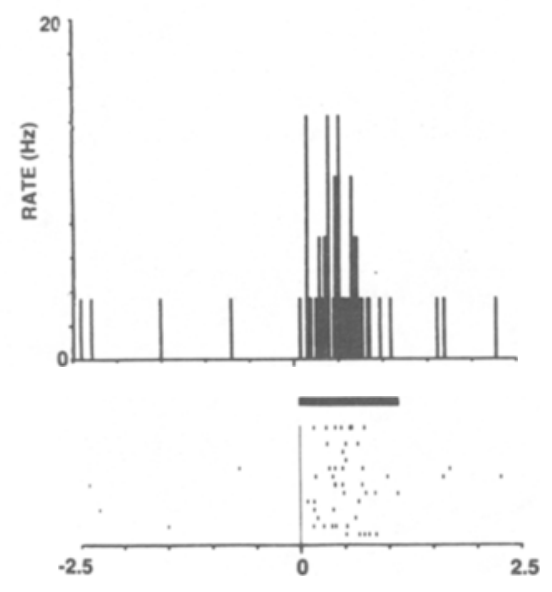

RIGHT TURNS
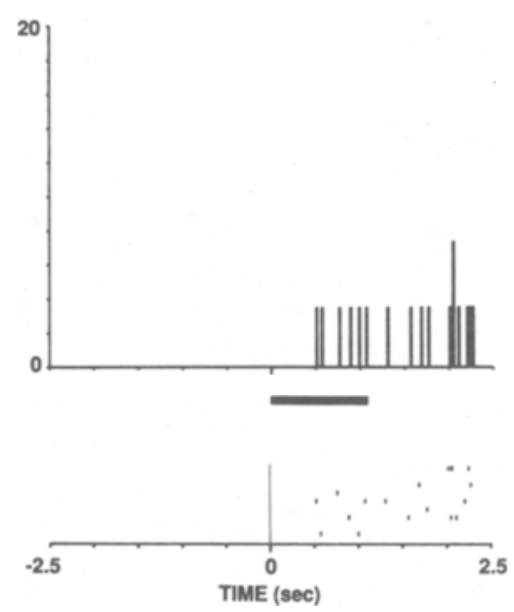

AT 3 EASTERN ARMS

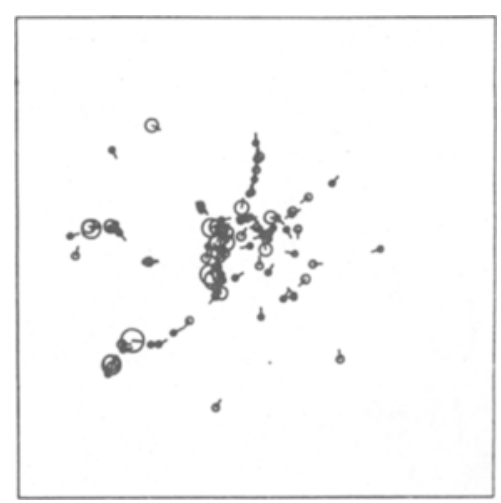

ALL LEFT TURNS

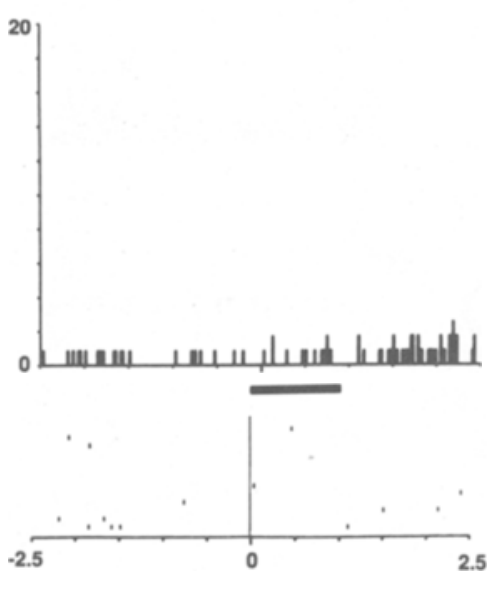

Figure 8. This parietal neuron was selectively active during right turns made at the ends of the west and southwest arms, and in the maze center when the animal was oriented in the same general direction. 
A
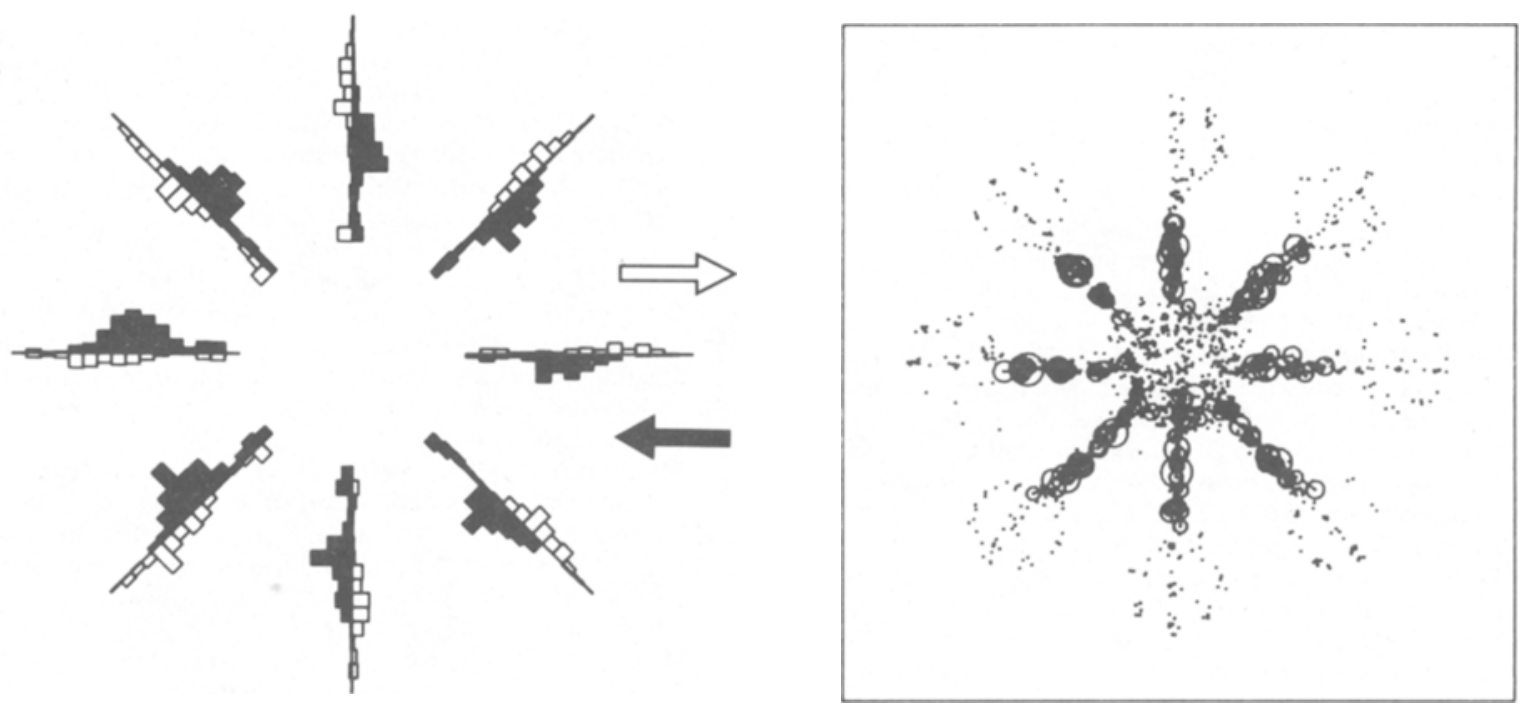

\section{B INWARD MOTION}

\section{OUTWARD MOTION}
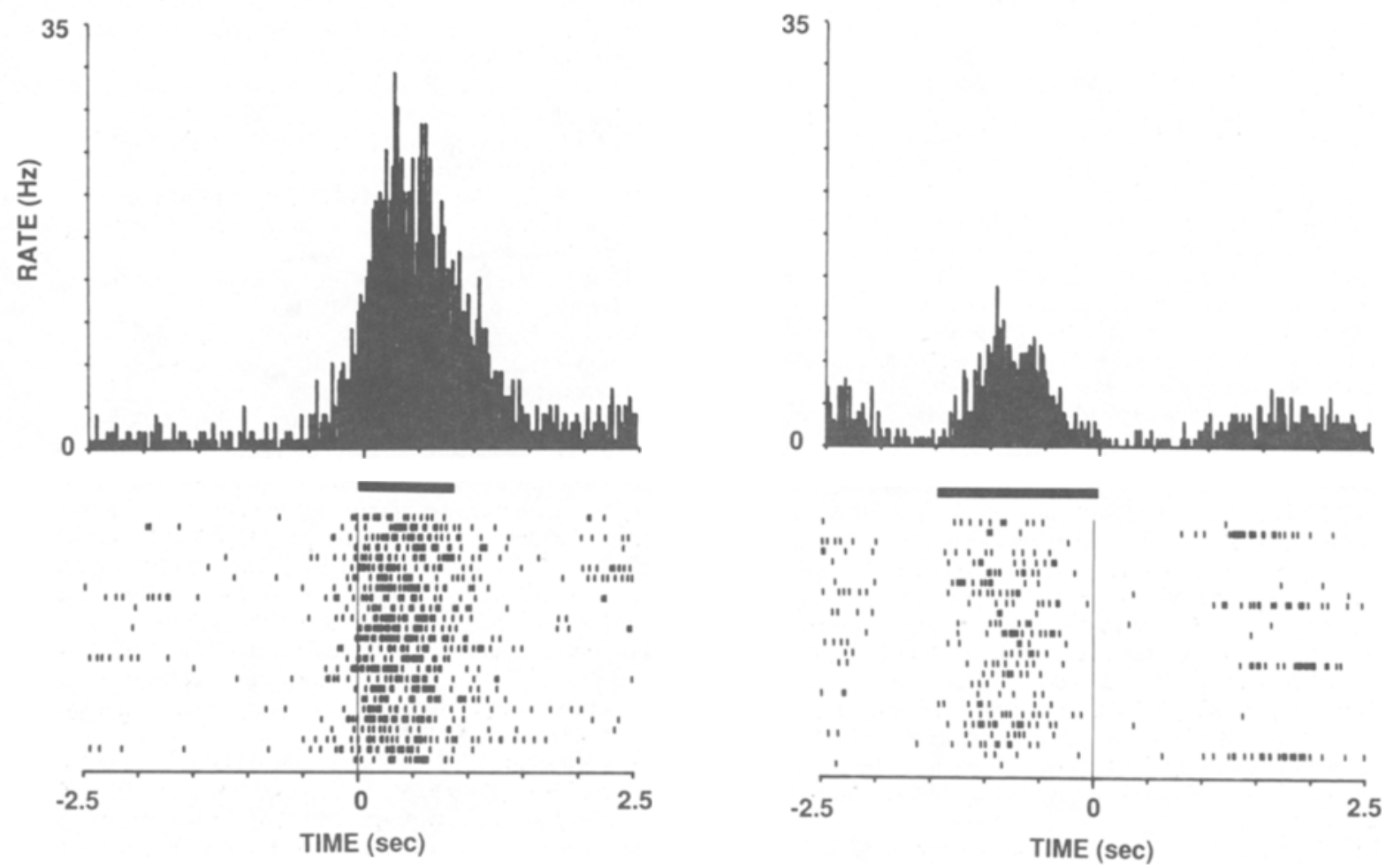

Figure 9. This parietal neuron was active generally during forward motion on the maze arms, and was inactive during turns. However, the activity was also conditional upon the radial direction of motion. The effect was not due to differential velocity. Moreover, forward motion through the maze center failed to activate the cell. 


\section{REFERENCES}

ANDERSEN, R. A. (1987). Inferior parietal lobule function in spatial perception and visuomotor integration. In V. B. Mountcastle, F. Plum, \& S. R. Geiger (Eds.), Handbook of physiology: The nervous system (Vol. 5, pp. 483-518). Bethesda, MD: American Physiological Society.

ANDersen, R. A., Essick, G. K., \& Siegel, R. M. (1985). Encoding of spatial location by posterior parietal neurons. Science, $230,456-458$.

BARnes, C. A. (1988). Spatial learning and memory processes: The search for their neurobiological mechanisms in the rat. Trends in Neurosciences, 11, 163-169.

Chen, L. L., \& McNaughton, B. L. (1988). Spatially selective discharge of vision and movement-modulated posterior parietal neurons in the rat. Society for Neuroscience Abstracts, 14, 818.

CoweY, A., Gross, C. G. (1979). Effects of foveal striate and inferotemporal lesions on visual discrimination by rhesus monkey. $E x$ perimental Brain Research, 11, 128-144.

Foster, T. C., Castro, C. A., \& McNaughton, B. L. (1988). Influence of motor set on hippocampal complex spike activity. Society for Neuroscience Abstracts, 14, 396.

Foster, T. C., Castro, C. A., McNaughton, B. L. (1989). Spatial selectivity of rat hippocampal neurons is dependent on preparedness for movement. Science, 244, 1580-1582.

Gross, C. G., Bruce, C. J., Desmoine, R., Fleming, J., \& GatTASs, R. (1981). Cortical visual areas of the temporal lobe. In C. N. Woolsey (Ed.), Cortical sensory organization: Vol. 2. Multiple visual areas (pp. 187-211). Clinton, NJ: Humana Press.

Hebs, D. O. (1949). The organization of behavior. New York: Wiley.

HiLl, A. J. (1978). First occurrence of hippocampal spatial firing in a new environment. Experimental Neurology, 62, 282-297.

HYVERINEN, J. (1982). The parietal cortex of monkey and man. Berlin: Springer-Verlag.

KoHONEN, T. (1972). Correlation matrix memories. IEEE Transactions on Computers, C-21, 353-359.

LASHLEY, K. S. (1932). The mechanism of vision: 5 . The structure and image-forming power of the rat's eye. Journal of Comparative Psychology, 13, 173-200.

Leonard, B., MCNaughton, B. L. (in press). Spatial representation in the rat: Conceptual, behavioral and neurophysiological perspectives. In R. Kesner \& D. S. Olton (Eds.), The neurobiology of comparative cognition. Hillsdale, $\mathrm{NJ}$ : Erlbaum.

Leonard, B. J., McNaughton, B. L., \& Barnes, C. A. (1985). Longterm studies of place field interrelationships in dentate gyrus neurons. Society for Neuroscience Abstracts, 11, 1108.

MARR, D. (1969). A theory of cerebellar cortex. Journal of Physiology, 202, 437-470.

MARR, D. (1971). Simple memory: A theory for archicortex. Philosophical Transactions of the Royal Society of London, B262, 23-81.

McNaughton, B. L. (1987). Neural association of movement and space: Preliminary steps toward a non-cartographic theory of spatial representation and learning. Neuroscience Letters, Supplement, 29, S143-S144.

McNaughton, B. L. (1989). Neuronal mechanisms for spatial computation and information storage. In L. Nadel, L. A. Cooper, P. Culicover, \& R. Harnish (Eds.), Neural connections and mental computations (pp. 285-349). Cambridge, MA: MIT Press.

McNaughton, B. L., Barnes, C. A., * O'Keefe, J. (1983). The contribution of position, direction and velocity to single unit activity in the hippocampus of freely-moving rats. Experimental Brain Research, 52, 41-49.

McNaughton, B. L., Green, E., \&izumori, S. J. Y. (1986). Representation of body-motion trajectory by rat sensory-motor cortex neurons. Society for Neuroscience Abstracts, 12, 260.
McNaughton, B. L., MorRis, R. G. M. (1987). Hippocampal synaptic enhancement and information storage within a distributed memory system. Trends in Neurosciences, 10, 408-415.

McNAughton, B. L., NADEL, L. (1989). Hebb-Marr networks and the neurobiological representation of action in space. In M. A. Gluck \& D. E. Rumelhart (Eds.), Neuroscience and connectionist theory (pp. 1-63). Hillsdale, NJ: Erlbaum.

McNaughton, B. L., O'Keefe, J., \& Barnes, C. A. (1983). The stereotrode: A new technique for simultaneous isolation of several single units in the central nervous system from multiple unit records. Journal of Neuroscience Methods, 8, 391-397.

Mishkin, M. (1982). A memory system in the monkey. Philosophical Transactions of the Royal Society of London, B298, 85-95.

Mishkin, M., Ungerleider, L. G., MAcko, K. A. (1983). Object vision and spatial vision: Two cortical pathways. Trends in Neurosciences, 6, 414-417.

Mittelstaedt, M. L., \& Mittelstaedt, H. (1980). Homing by path integration in a mammal. Naturwissenschafien, 67S, 566 .

Motrer, B. C., MountCastle, V. B. (1981). The functional properties of light-sensitive neurons of the posterior parietal cortex studied in waking monkeys: Foveal sparing and opponent vector organization. Journal of Neuroscience, 1, 3-26.

Motter, B. C., Steinmetz, M. A., Duffy, C. J., \& Mountcastle, V. B. (1987). Functional properties of parietal visual neurons: Mechanisms of directionality along a single axis. Journal of Neuroscience, 7, 154-176.

Mountcastle, V. B., Lynch, J. C., Georgopoulos, A., Sakata, H., \& ACUNA, C. (1975). Posterior parietal association cortex in the monkey: Command functions for operations within extrapersonal space. Journal of Neurophysiology, 38, 871-908.

Muller, R. U., KUBIE, J. L., \& RANCK, J. B., JR. (1987). Spatial firing patterns of hippocampal complex-spike cells in fixed environment. Joumal of Neuroscience, 7, 1935-1950.

O'KeEFE, J. (1976). Place units in the hippocampus of the freely moving rat. Experimental Neurology, 51, 78-109.

OKREFE, J., CoNWAY, D. H. (1980). On the trail of the hippocampal engram. Physiological Psychology, 8, 229-238.

O'KeEFE, J., Dostrovsky, J. (1971). The hippocampus as a spatial map: Preliminary evidence from unit activity in the freely-moving rat. Brain Research, 34, 171-175.

O'KEEFE, J., \& NADEL, L. (1978). The hippocampus as a cognitive map. Oxford: Oxford University Press.

O'KeEFE, J., SPEAKMAN, A. (1987). Single unit activity in the rat hippocampus during a spatial memory task. Experimental Brain Research, 68, 1-27.

Olton, D. S., Branch, M., \& Best, P. J. (1978). Spatial correlates of hippocampal unit activity. Experimental Neurology, 58, 387-409.

Sakata, H., Shibutani, H., Kawano, K., \& Harrington, T. (1985). Neural mechanisms of space vision in the parietal association cortex of the monkey. Vision Research, 25, 453-464.

SMITH, M. L., MILNER, B. (1981). The role of the right hippocampus in the recall of spatial location. Neuropsychologia, 19, 781-793.

SteinBuCH, K. (1961). Die lernmatrix. Kybernetik, 1, 36-45.

Tanaka, K., Hikosaka, K., Saito, H., Yukie, M., Fukada, Y., \& IWAI, E. (1986). Analysis of local and wide-field movements in the superior temporal visual areas of the macaque monkey. Journal of Neuroscience, 6, 134-144.

UNGerLeIDER, L. G., \&ishrin, M. (1982). Two cortical visual systems. In D. J. Ingle, M. A. Goodale, \& R. J. Wansfield (Eds.), Analysis of visual behavior (pp. 549-586). Cambridge, MA: MIT Press.

Willshaw, D. J., Buneman, O. P., Longuet-Higgins, H. C. (1969). Non-holographic associative memory. Nature, 222, 960962. 